\title{
Effect of active species in crude oil on the interfacial tension behavior of alkali/synthetic surfactants/ crude oil systems
}

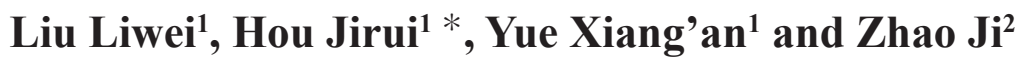 \\ ${ }^{1}$ Key Laboratory of Petroleum Engineering, Ministry of Education, China University of Petroleum, Beijing 102249, China \\ ${ }^{2}$ China Petrochemical Corporation, Beijing 100029, China
}

\begin{abstract}
The effect of active species present in crude oil on the interfacial tension (IFT) behavior of alkali/synthetic surfactants/crude oil systems was studied. The system consisted of heavy alkyl benzene sulfonate, sodium chloride, sodium hydrate and Daqing crude oil. Experimental results indicated that active species would diffuse from oil/aqueous interface to aqueous phase and finally an equilibrium could be reached in the system with increasing contact time. Moreover, the minimum IFT and equilibrium IFT values increased with increasing contact time and a linear relationship existed between dynamic IFT and $t^{-1 / 2}$ when IFT value approaching the minimum and after the minimum IFT was reached. This indicated that the dynamic IFT-time behavior was diffusion controlled. The oil and aqueous phases were analyzed by infrared (IR) spectroscopy. IR spectra of oil and aqueous phases illustrated that the content of active species in the oil phase decreased, but the content of active species in the aqueous phase increased after alkali reacted with crude oil. This indicated that the active species present in oil played an important role in reducing IFT.
\end{abstract}

Key words: Contact time, diffusion, alkali, interfacial tension

\section{Introduction}

Alkali/surfactant/polymer (ASP) flooding, a new technology for enhanced oil recovery, has been applied in China and overseas in recent years (Liu et al, 2007b; Touhami et al, 2001; Zhao et al, 2006), and oil recovery could be further enhanced by $20 \%$ by ASP flooding for heterogeneous reservoirs. ASP flooding embodies the characteristics of alkaline flooding, surfactant flooding and polymer flooding, and in ASP flooding synergistic effect of alkali, surfactant, and polymer makes ASP flooding effective (Chen et al, 2001; Cheng and Zhang, 2004; Feng et al, 2001). The active species in crude oil react with alkali and generate surfaceactive species which play a very important role in reducing interfacial tension (Chan and Yen, 1982; Luo et al 2004; Zhang et al, 2001)

At present, the method commonly used to study active species in crude oil is extraction, in which organic acids and oxygen-containing components are extracted from crude oil (Luo et al, 2000). However, in the actual displacement process displacing fluid will contact with crude oil and react with some active species in the crude oil (Liu et al, 2007a). This process may be continuing for a long time, and contact time may play an important role in reducing interfacial tension. It is essential to investigate systematically how

*Corresponding author. email: houjirui@126.com Received January 22, 2008
IFT changes with contact time. In this paper, the concept of contact time was introduced to investigate the influence of active species in crude oil on the IFT, and the migration of active species.

\section{Experimental}

\subsection{Materials}

Heavy alkyl benzene sulfonate (HABS), used as a surfactant, was provided by Daqing Oilfield Research Institute. HABS is a viscous liquid at room temperature, with an average molecular weight of 433 and an active matter content of $50 \%$. The molecular structural formula is:

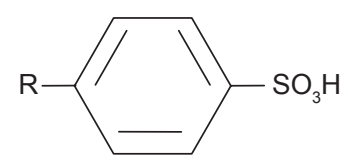

where $\mathrm{R}$ is alkyl group with a chain length of $\mathrm{C}_{12}-\mathrm{C}_{18}$.

$\mathrm{NaOH}$ and $\mathrm{NaCl}$ are commercial analytical reagents (Beijing Chemical Reagents Company). Straight-run kerosene, provided by the State Key Laboratory of Heavy Oil Processing, China University of Petroleum (Beijing), has a density of $0.7809 \mathrm{~g} / \mathrm{m}^{3}$. The water used in all experiments is distilled water.

Daqing crude oil was obtained from the First Oil Production Factory, Daqing Oilfield. The oil samples were 
degassed and dewatered before use. The total acid number of the crude oil was determined by using ASTM method D664. Daqing crude oil has a acid number of $0.06 \mathrm{mg} \mathrm{KOH} / \mathrm{g}$ oil, a density of $0.96075 \mathrm{~g} / \mathrm{m}^{3}$, and a viscosity of $9.80 \mathrm{mPa} \cdot \mathrm{s}$ at $25^{\circ} \mathrm{C}$. This indicates that Daqing crude oil has a small amount of organic acids.

Active-species-enriched system: $30 \mathrm{ml}$ of Daqing crude oil were added into a beaker containing $60 \mathrm{ml}$ mixed solution (The mixed solution contained $1.5 \% \mathrm{NaCl}$ and $0.3 \% \mathrm{NaOH}$ ). After stirring evenly (avoiding emulsification) (Guo et al, 2006; Li et al, 2004), the mixture stood for $24 \mathrm{~h}$ at room temperature. Then $20 \mathrm{ml}$ of the mixture were taken out with a microsyringe from the beaker and then filtered (Buchner filter). Finally $4 \mathrm{ml}$ of HABS were added to the mixed solution of $20 \mathrm{ml}$. The as-prepared solution was named as active-species-enriched system.

\subsection{Apparatus}

A spinning drop interfacial tensiometer (Model 500, Temoco Inc.) was used to perform the ITF measurements. A Shimadzu AY120 analytical balance was used to weigh chemicals. Active species in crude oil were characterized with a Nicolet Nexus 670 Fourier transform infrared spectrometer (FT-IR).

\subsection{Experimental procedure}

With a microsyringe, oil was injected into a glass tube $(\Phi=5 \mathrm{~mm})$ filled with surfactant phase (a mixture of $1.5 \%$ $\mathrm{NaCl}, 0.3 \% \mathrm{NaOH}, 0.2 \% \mathrm{HABS}$ ). The volume of the oil injected was about $2 \mu \mathrm{l}$ and the volume of the glass tube was about $0.29 \mathrm{ml}$. In experiments, the oil droplet injected should contacted with the surfactant phase well. Then the tube was placed in an incubator at $45{ }^{\circ} \mathrm{C}$. After a specified pre-contact time (from 0 to 1440 minutes), the tube was taken out, and the dynamic IFT was measured by the interfacial tensiometer.

\section{Results and discussion}

\subsection{Effect of active species on interfacial tension of oil/alkali/HABS systems}

Fig. 1 shows the dynamic IFT-time behavior of Daqing crude oil/alkali/HABS systems. For crude oil/alkali/HABS systems, the minimum IFT value of less than $0.01 \mathrm{mN} / \mathrm{m}$, obviously below the equilibrium value, could be obtained when the pre-contact time was 0-60 min. However, when the pre-contact time exceeded $120 \mathrm{~min}$, the minimum IFT value would be higher than $0.01 \mathrm{mN} / \mathrm{m}$, close to the equilibrium value of this system. With increasing pre-contact time, the ratio of the minimum IFT to the equilibrium increased gradually and approximated 1 (Table 1).

When straight-run kerosene, without containing any acidic components, was used as oil phase, different experimental results were obtained under the same experimental conditions. Fig. 2 shows that the pre-contact time had a slight effect on dynamic IFT-time behavior of straight-run kerosene/alkali/ HABS systems, and minimum IFT value was close to the equilibrium value for these systems, independent of the precontact time.

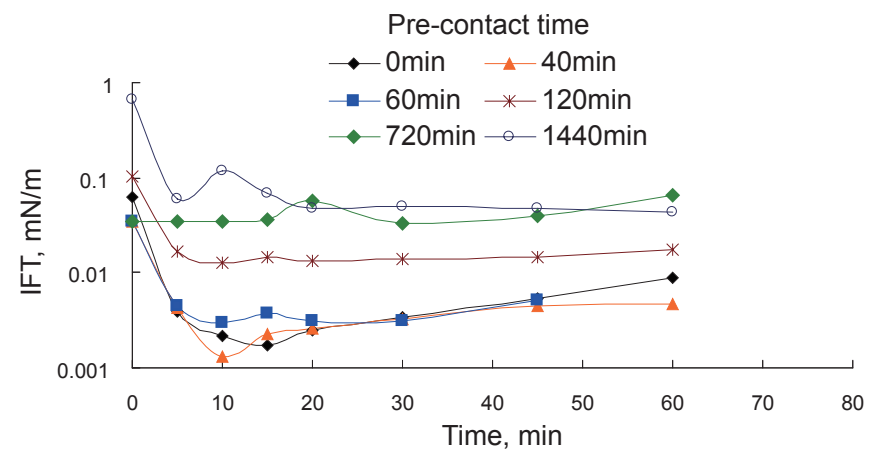

Fig. 1 Dynamic IFT-time behavior of crude oil/alkali/HABS systems

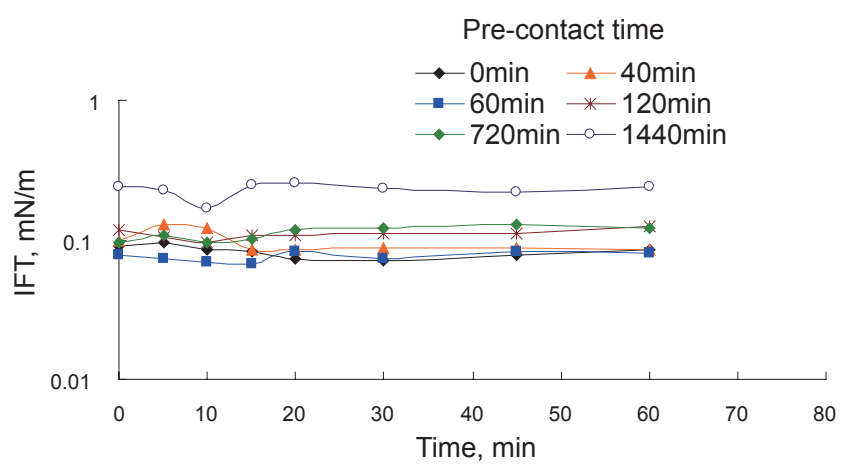

Fig. 2 Dynamic IFT-time behavior of straight-run kerosene/ alkali/HABS systems

Figs. 1 and 2 show that there existed a noticeable difference in IFT behavior of crude oil/alkali/HABS systems and kerosene/alkali/HABS systems. Possible explanations for the above-mentioned phenomenon are that 1) Active species present in crude oil can migrate to the interface, where they react with alkali from the aqueous phase to form selfgenerated surface-active species. The surface-active species play an importance role in reducing IFT. 2) Surface-active species formed on the interface can migrate into the bulk aqueous phase with prolonging contact time. The migration of self-generated surface-active species from interface to aqueous phase increases the concentration of surface-active species in the bulk aqueous phase, and decreases those absorbed on the interface. When both concentrations become the same, the IFT reaches an equilibrium value. This process is corresponding to IFT-time behavior.

Table 1 Ratio of minimum IFT to equilibrium IFT

\begin{tabular}{cccc}
\hline $\begin{array}{c}\text { Pre-contact time } \\
\text { min }\end{array}$ & $\begin{array}{c}\text { Minimum IFT } \\
\mathrm{mN} / \mathrm{m}\end{array}$ & $\begin{array}{c}\text { Equilibrium IFT } \\
\mathrm{N} / \mathrm{m}\end{array}$ & Ratio \\
\hline 0 & $1.7 \times 10^{-3}$ & $5.4 \times 10^{-3}$ & 0.31 \\
40 & $1.3 \times 10^{-3}$ & $4.7 \times 10^{-3}$ & 0.28 \\
60 & $3.0 \times 10^{-3}$ & $5.2 \times 10^{-3}$ & 0.58 \\
120 & $1.3 \times 10^{-2}$ & $1.7 \times 10^{-2}$ & 0.76 \\
720 & $3.4 \times 10^{-2}$ & $3.9 \times 10^{-2}$ & 0.87 \\
1440 & $4.8 \times 10^{-2}$ & $4.8 \times 10^{-2}$ & 1.00 \\
\hline
\end{tabular}




\subsection{Detection of active species in crude oil}

In order to analyze the function of the active species in crude oil, an active-species-enriched system was prepared. The IFT behavior of crude oil/ active-species-enriched system was measured, and shown in Fig. 3. For comparison, dynamic IFT of crude oil/alkali/HABS system (normal system) was also shown in Fig. 3.

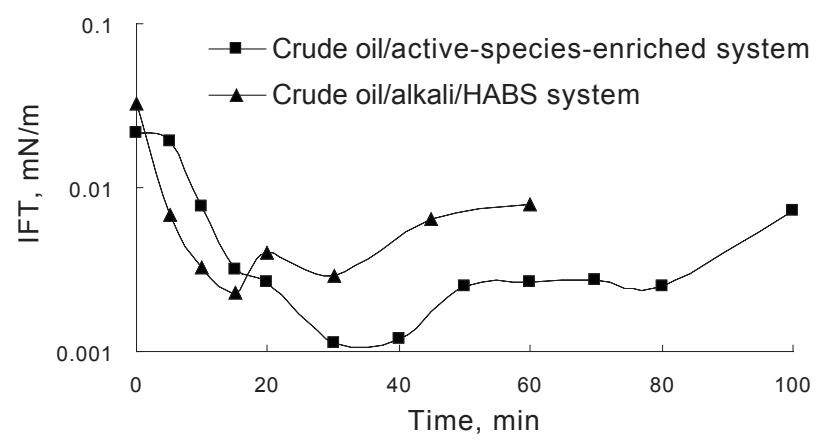

Fig 3 Dynamic IFT-time behavior the crude oil/active-speciesenriched system and crude oil/alkali/HABS system (The pre-contact time was $0 \mathrm{~min}$ )

In the case of crude oil in contact with the active-speciesenriched system, the ultra low interfacial tension (less than $10^{-3} \mathrm{mN} / \mathrm{m}$ ) was reached and remained at the ultra low range for a long period of time. Moreover, the minimum value and equilibrium value were significantly lower than those of crude oil in contact with alkali/HABS solution. A possible explanation is that there exist plenty of self-generated surface-active species, formed by reaction of $\mathrm{NaOH}$ with active species present in crude oil in advance, in the so-called active-species-enriched system. Therefore, the concentration of surface-active species in the case of crude oil in contact with the active-species-enriched system is much higher than that in the normal system. The surface-active species can be strongly adsorbed on the oil/water interface, which to a large extent, helps surfactant added later to reduce dynamic IFT and to maintain the minimum IFT value for a long period of time.

Figs. 4 and 5 show infrared spectra of Daqing crude oil and crude oil in full contact with the displacing fluid, respectively. The absorption peaks of organic acid at 1,699 (carbonyl stretching vibration), 1,602 (carboxylate), and $2,672 \mathrm{~cm}^{-1}$ (carboxylic acid) were weakened after crude oil contacted fully with displacing fluid. The ratio of absorption strength of stretching vibration of $\mathrm{C}=\mathrm{O}$ at $1,701.50 \mathrm{~cm}^{-1}$ (or $1,669 \mathrm{~cm}^{-1}$ ) to that of deformation vibration of methylene at $1,462.37 \mathrm{~cm}^{-1}$ can reflect the change of organic acid in oil droplet. For the Daqing crude oil sample, its value was 0.077 $(0.5 / 6.5=0.077)$; for the oil droplet that contacted well with the displacing fluid, its value was $0.041(0.2 / 4.9=0.041)$. Experimental results showed that the content of organic acid in crude oil decreased after contact with the displacing fluid. Moreover, the stretching peak of the carboxylic ion at 1,576 $\mathrm{cm}^{-1}$ was detected in the displacing fluid phase of the crude oil/displacing fluid (Fig. 6), which is a measure of the degree of formation of petroleum soap. After contact with the crude oil sample, the displacing fluid was extracted with acetone and some surface-active species formed in the displacing fluid could be extracted out with acetone. In the IR scanning of the extract, organic acid salt and phenolics were detected (Fig. 7). This indicates that the active species present in crude oil play a key role in reducing the IFT.

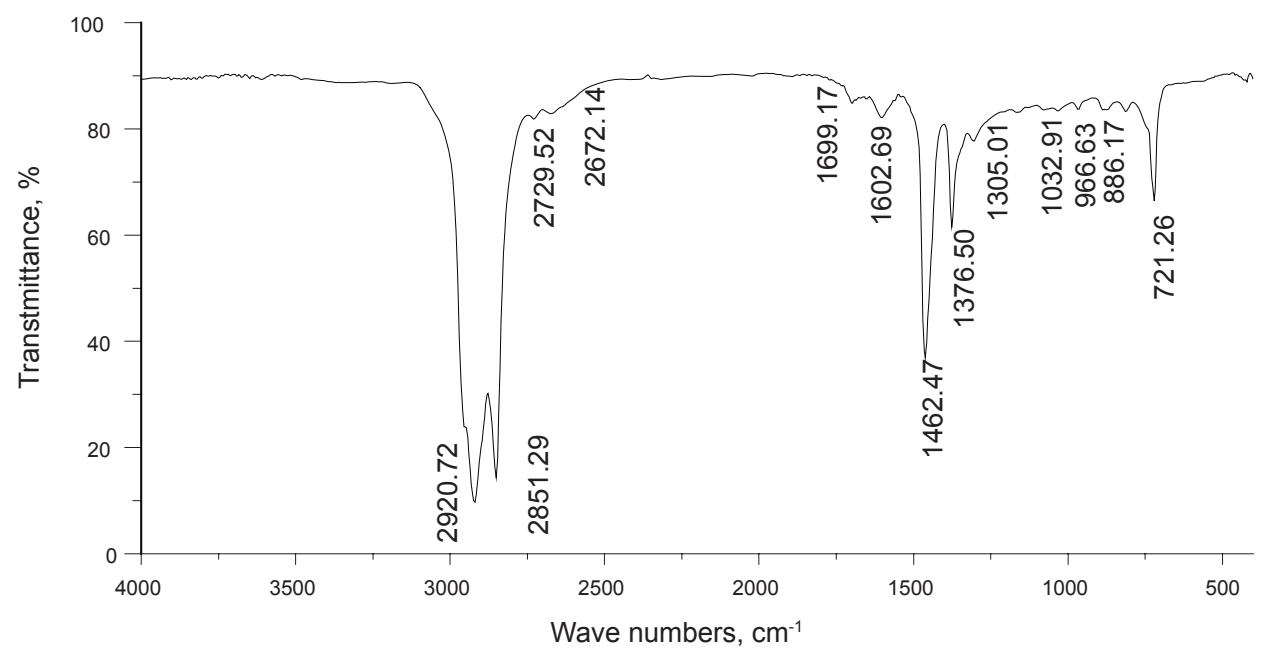

Fig. 4 IR spectrum of the Daqing crude oil sample

\subsection{Diffusion of surface-active species in oil/alkali/ HABS systems}

Several important factors: temperature, ionic strength of aqueous phase and viscosity of the oil and aqueous phases, affect the diffusion of surface-active species in the oil/alkali/
HABS system. The temperature and viscosity (both oil and aqueous phases) were constant in these experiments, while the ionic strength was affected primarily by the sodium hydroxide concentration. In 1960, Bird and coworkers (2002) proposed the Stokes-Einstein equation, i.e., diffusion coefficient is related to solution viscosity. 


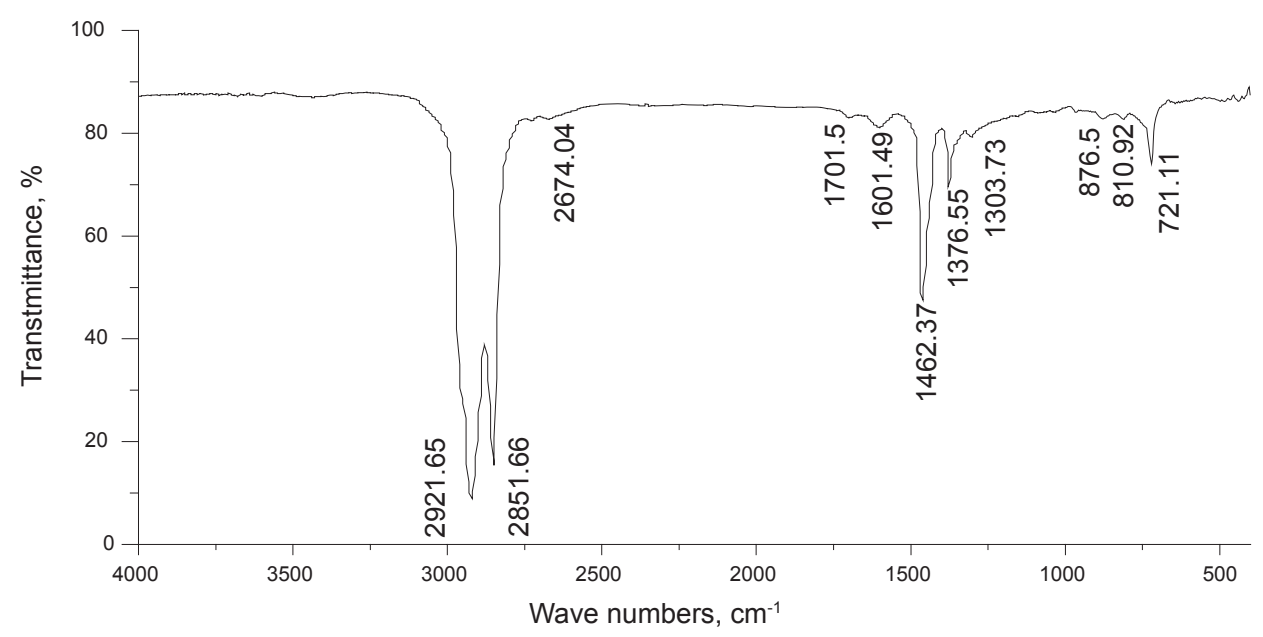

Fig. 5 IR spectrum of crude oil sample after $1440 \mathrm{~min}$ of contact with displacing fluid

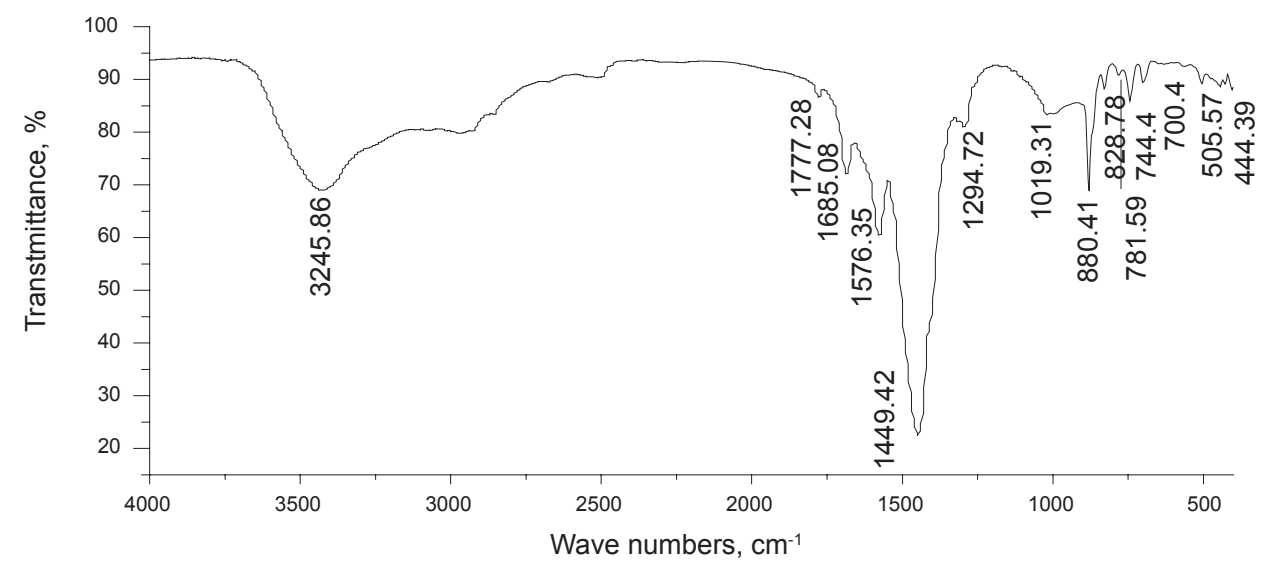

Fig. 6 IR spectrum of displacing fluid phase of the crude oil/displacing fluid system

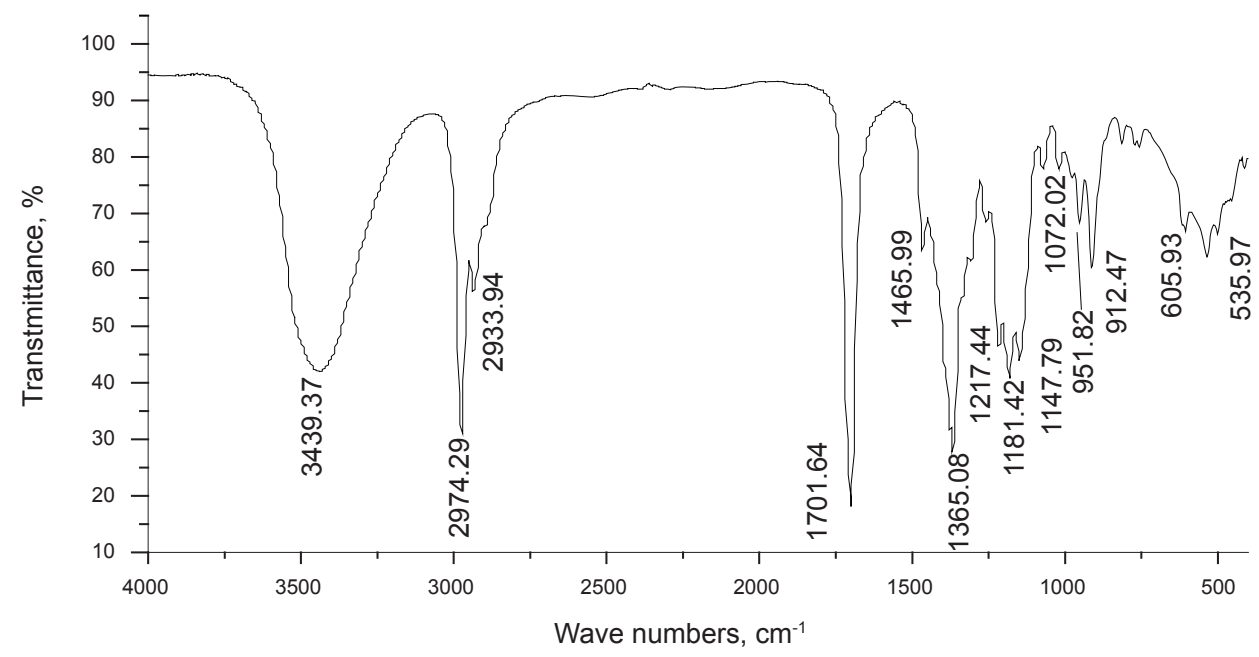

Fig. 7 IR spectrum of the surface-active species formed in the displacing fluid

$$
D=\frac{k T}{6 \pi \mu_{n}} r
$$

where $D$ is the diffusion coefficient, the $\mu_{n}$ is the viscosity of the medium, $k$ is Boltzmann's constant, and $r$ is the radius of the molecule.
The temperature and viscosity of the oil/alkali/HABS system are constant, so $D$ is constant too. According to van Hunsel and Joos (1989):

$$
\operatorname{IFT}(t)=\operatorname{IFT}_{\mathrm{e}}+\frac{R T \Gamma^{2}}{C_{\mathrm{o}}}\left(\frac{\pi}{4 D t}\right)^{\frac{1}{2}}
$$


where $\operatorname{IFT}(t)$ is interfacial tension as a function of time, $t$; $\mathrm{IFT}_{\mathrm{e}}$ is the equilibrium IFT; $C_{\mathrm{o}}$ is the initial surfactant concentration; and $\Gamma$ is the adsorption on the interface. For oil/alkali/HABS systems, $C_{\mathrm{o}}$ and $\Gamma$ will vary with alkali concentration, among other things (Taylor and Nasr-EL-Din, 1996). If IFT is plotted versus $t^{-1 / 2}$, a straight line signifies that the process is diffusion controlled.

Table 2 summarizes the slope (inversely proportional to $D^{-1 / 2}$ ) and the intercept obtained in each case, where IFT was plotted versus time before and after the minimum IFT was reached. Fig. 8 shows the linear relationship between IFT and $t^{-1 / 2}$ for oil/alkali/HABS systems $(1.5 \% \mathrm{NaCl}, 0.3 \% \mathrm{NaOH}$, and $0.2 \%$ HABS ). It can clearly be seen that the slope of the line increases, but the intercept of the line decreases with the pre-contact time before the minimum IFT is reached. Fig. 9 shows that after the minimum IFT is reached, the slope of the line decreases, but the intercept of the line increases with the pre-contact time.

Table 2 Regression data, IFT versus $t^{-1 / 2}$

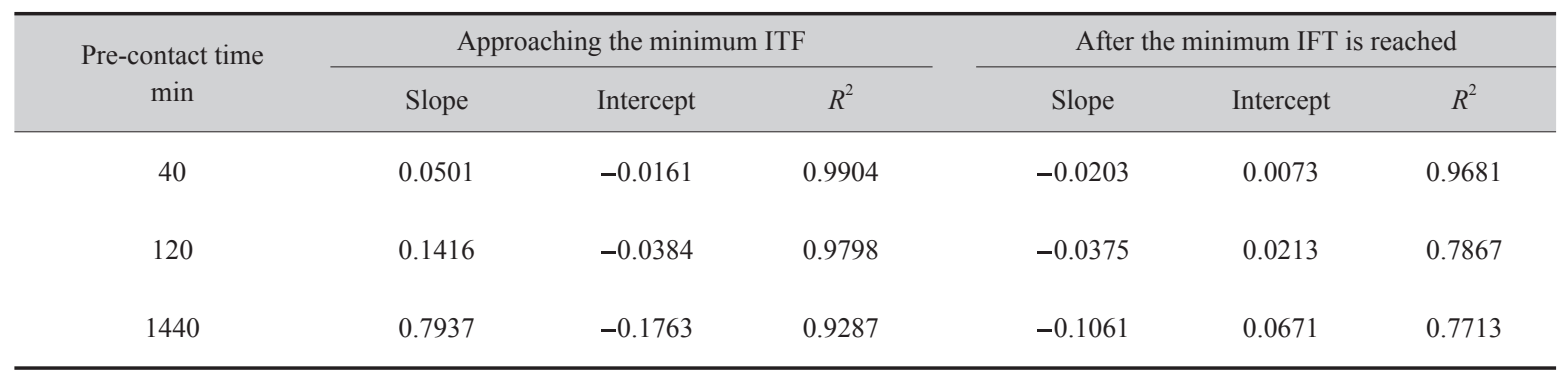

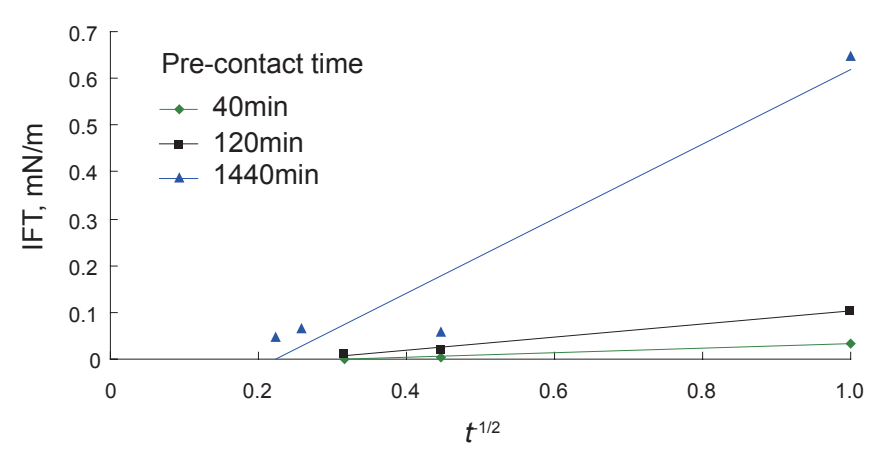

Fig. 8 IFT versus $t^{-1 / 2}$, approaching the minimum IFT

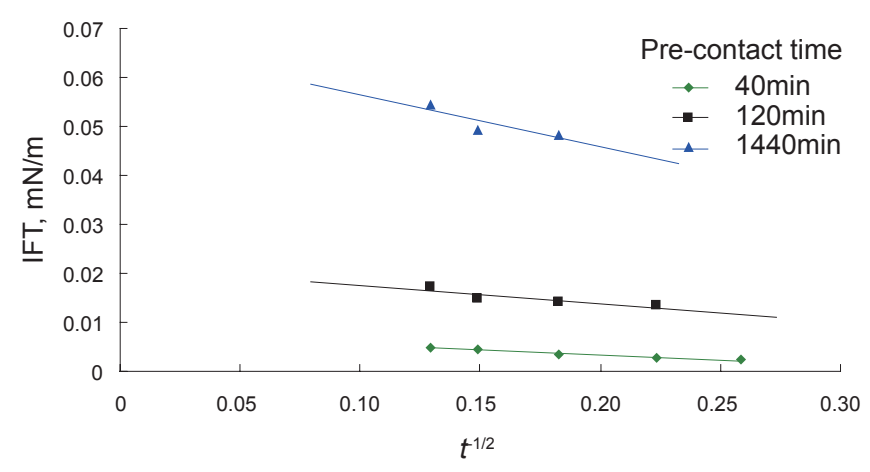

Fig. 9 IFT versus $t^{-1 / 2}$, after the minimum IFT is reached

Figs. 8 and 9 indicate that the dynamic IFT-time behavior is diffusion controlled. The acidic active species present in crude oil react with alkali to form self-generated surfaceactive species, then the surface-active species are adsorbed on the interface between oil and aqueous phases. When the concentration of surface-active species adsorbed on interface reaches a maximum value, they begin to migrate to the aqueous phase. The correction coefficient $R^{2}$ decreases with the contact time. This phenomenon indicates that diffusion process diminishes and finally cease. Finally the surfactant concentration is uniform in the whole compound system and an equilibrium IFT can be reached in the system.

\section{Conclusions}

1) With increasing pre-contact time, the minimum IFT of crude oil/alkali/HABS systems increases quickly, but the equilibrium IFT increases slowly. Therefore, the ratio of them increases gradually and finally reaches approximately 1. The reason for this phenomenon may be the consumption of active species present in crude oil and diffusion of selfgenerated surface-active species from oil/aqueous interface to aqueous phase.

2) Alkali can react with the active species present in crude oil to form surface-active species in situ. This surface-active species in crude oil play an important role in reducing IFT.

3) Before and after minimum IFT is reached, a linear relationship exists between IFT and $t^{-1 / 2}$. This indicates that the dynamic IFT-time behavior process is diffusion controlled.

\section{Acknowledgements}

This work was financially supported by National Basic Research Program of China (973 Program). The authors would like to thank them for their approval to publish this paper.

\section{References}

Bird P B, Stewart W E and Lightfoot E M. Transport Phenomena. New York: John Wiley Press. 2002

Chan $\mathrm{M}$ and Yen T F. A chemical equilibrium model for interfacial activity of crude oil in aqueous alkaline solution. Canada J Chem. Eng. 1982. 60: 305-308

Chen Y M, Wang H H and Yu J Y. Research advance of dynamic interfacial tension behavior in chemical flooding. Acta Petrolei Sinica. 2001. 22 (4): 97-103 (in Chinese) 
Cheng J C and Zhang Y X. Effect of $\mathrm{Na}_{2} \mathrm{CO}_{3} /$ alkyl benzene sulfonate/ polymer system on interfacial tension between oil and water. Journal of Daqing Petroleum Institute. 2004. 28 (3): 37-39 (in Chinese)

Feng L, Li Z S and Qiao W H. Study of dynamic and equilibrium interfacial tension of alkali/alkyl sulfates and alkali/alkanesulfonates solution with Daqing crude oil. Fine Chemicals. 2001. 18 (1): 18-20 (in Chinese)

Guo J X, Liu Q, Li M Y, et al. The effect of alkali on crude oil/water interfacial properties and the stability of crude oil emulsions. Colloids and Surfaces A: Physicochemical and Engineering Aspects. 2006. 273: $213-218$

Li M Y, Lin M Q, Wu. Z L, et al. The influence of $\mathrm{NaOH}$ on the stability of paraffinic crude oil emulsion. Fuel. 2004. 84: 183-187

Liu L W, Hou J R and Yue X A. The influence of pre-contact time on the interfacial tension in combinative system. Journal of Southwest Petroleum University. 2007a. 29 (5): 105-107 (in Chinese)

Liu Q, Dong. M Z, Ma S Z, et al. Surfactant enhanced alkaline flooding for Western Canadian heavy oil recovery. Colloids and Surfaces A: Physicochemical and Engineering Aspects. 2007b. 293: 63-71

Luo L, Zhang L and Fang H B. Effect of EOR chemicals on interfacial dilational properties of active constituents in crude oil. Petroleum
Exploration and Development. 2004. 31: 21-25 (in Chinese)

Luo L, Zhao S and Zhang L. Separation strucutral analysis interfacial activity of acidic components in Liaohe crude oil. Oilfield Chemistry. 2000. 17(2): 152-155 (in Chinese)

Taylor K C and Nasr-EL-Din H A. The effect of synthetic surfactants on the interfacial behavior of crude oil/alkali/polymer systems. Colloids and Surfaces A: Physicochemical and Engineering Aspects. 1996. 108: 49-72

Touhami Y, Rana D, Hornof V, et al. Effects of added surfactant on the dynamic interfacial tension behavior of acidic oil/alkaline systems. Journal of Colloid and Interface Science. 2001. 239: 226-229

van Hunsel J and Joos P. Study of the dynamic interfacial tension at the oil/water interface. Colloid Polymer Science. 1989. 267: 1026-1035

Zhang L, Luo L and Li Z Q. Dynamic interfacial tension between Shengli crude oil and different enhanced oil recovery systems. Oilfield Chemistry. 2001. 18(1): 67-70 (in Chinese)

Zhao Z K, Bi C G, Li Z S and Qiao W H. Interfacial tension between crude oil and decylmethylnaphthalene sulfonate surfactant alkalifree flooding systems. Colloids and Surfaces A: Physicochemical and Engineering Aspects. 2006. 276: 186

(Edited by Sun Yanhua) 